\title{
XAFS and XMCD Spectra at the Surface and Interface of Ultrathin Films Observed by the Depth-Resolved XAFS/XMCD Technique*
}

\author{
Kenta Amemiya ${ }^{\dagger}$ and Masako Sakamaki \\ Institute of Materials Structure Science, High Energy Accelerator \\ Research Organization, 1-1 Oho, Tsukuba-shi, Ibaraki, 305-0801, Japan \\ (Received 10 August 2012; Accepted 5 September 2012; Published 22 September 2012)
}

\begin{abstract}
A depth-resolved X-ray absorption fine structure (XAFS) technique has been developed by using the detectionangle dependence of the effective escape depth of the Auger electrons in the electron-yield XAFS measurement. The technique is applied to a Ni thin film covered with oxygen adsorbates in order to extract the XAFS and X-ray magnetic circular dichroism (XMCD) spectra at the surface layer. The chemical state and the magnetic structure of the surface layer are clarified from the extracted XAFS/XMCD spectra. [DOI: 10.1380/ejssnt.2012.521]
\end{abstract}

Keywords: X-ray Absorption Fine Structure (XAFS); X-ray Magnetic Circular Dichroism (XMCD); Depth-Resolved Technique; Surface and Interface; Magnetic Thin Films

\section{INTRODUCTION}

The depth profile of magnetic ultrathin films has attracted much interest because the surface and interface play essential roles in the magnetic property of the whole film. The X-ray magnetic circular dichroism (XMCD) technique is one of the most powerful tools to investigate magnetic materials, by which the spin and orbital magnetic moments are element-specifically determined $[1,2]$, but the conventional XMCD measurement has no depth resolution. To separately obtain information on the surface and interface magnetic structures, a depthresolved XMCD technique has been developed, which has an atomic-layer resolution [3-5]. Of course, the depthresolved technique is also applicable to X-ray absorption fine structure (XAFS) measurements in general.

In the present paper, an example for the observation of the surface XAFS/XMCD spectra is presented for an oxygen-covered $\mathrm{Ni}$ film [6] after a brief introduction to the depth-resolved XAFS/XMCD technique. (a) Concept

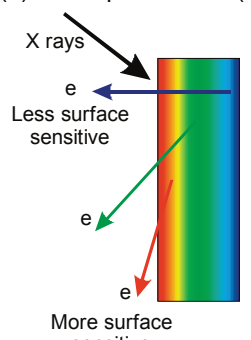

sensitive (b) Normal incidence

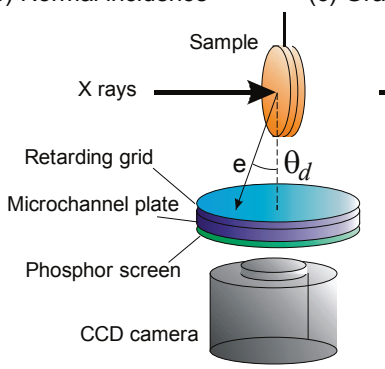

(c) Grazing incidence

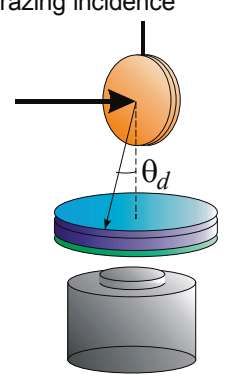

FIG. 1: Schematic concept of the depth-resolved XAFS technique (a) and experimental setup with an imaging-type detector consisting of a microchannel plate, a phosphor screen and a CCD camera, in the normal (b) and grazing (c) X-ray incidence configurations. The probing depth is determined by the electron detection angle, $\theta_{\mathrm{d}}$.

\footnotetext{
* This paper was presented at the conference "XAFS theory and nano particles", Chiba University, Chiba, Japan, July 18-20, 2012. †Corresponding author: kenta.amemiya@kek.jp
}

\section{PRINCIPLE OF THE TECHNIQUE}

The depth-resolved XAFS/XMCD technique is based on the well-known electron-yield XAFS/XMCD measurements, in which the electrons emitted after the X-ray absorption process are collected to obtain the absorption spectra. The electrons generated in a solid sample undergo the attenuation before they reach the surface and then collected by a detector. The effective escape depth for the electrons strongly depends on the electron emission angles, as shown in Fig. 1(a), because the electron path length is longer at more grazing emission angle. Therefore, to realize the depth resolution, a series of spectra with different probing depths are recorded by separately collecting the electrons at different detection angles, $\theta_{\mathrm{d}}$, by using an imaging-type detector, as shown in Figs. 1(b) and $1(\mathrm{c})$. Note here that any X-ray incidence angle is available, because the depth resolution is achieved mainly by the attenuation of the emitted electrons. One can extract the depth-resolved XAFS/XMCD spectra for ultrathin films with an atomic-layer resolution, by analyzing a set of spectra with different probing depths.

We define the effective escape depth of the electrons as $\lambda_{\mathrm{e}}$, and assume that the electrons generated at a depth $z$ are attenuated by a factor, $\exp \left(-z / \lambda_{\mathrm{e}}\right)$, before they are detected. The attenuation of the incident $\mathrm{X}$ rays must be also considered, though the effect of the attenuation of the $\mathrm{X}$ rays is much smaller than that of the electrons. The observed X-ray absorption data after the background subtraction and the normalization to the edge-jump intensity is given by

$Y(E)=C \sum_{i=1}^{n} \mu_{i}(E) \exp \left[-d\left\{\frac{i-1}{\lambda_{\mathrm{e}}}+\frac{1}{\cos \theta} \sum_{k=1}^{i-1} \mu_{k}(E)\right\}\right]$,

where $C$ is the normalization factor, $n$ the film thickness in a ML unit, $\mu_{i}(E)$ the absorption coefficient at the $i$ th layer from the top layer, $d$ the layer spacing, $E$ the photon energy and $\theta$ the X-ray incidence angle from surface normal. The first and second terms in the exponential function correspond to the attenuations of the electrons and incident $\mathrm{X}$ rays, respectively. Since $C$ is independent of the photon energy, it is determined at each $\lambda_{\mathrm{e}}$, by using the known absorption coefficients at the pre- and post-edge energies. Then $\mu_{i}(E)$ values are optimized so as to reproduce the observed set of $Y(E)$ values at dif- 
ferent $\lambda_{\mathrm{e}}$, resulting in the layer-resolved X-ray absorption spectra.

\section{SAMPLE PREPARATION AND MEASUREMENT}

All of our experiments were performed in an ultrahighvacuum chamber placed at soft X-ray beamlines, BL-7A, 11A, and 16A of the Photon Factory in High Energy Accelerator Research Organization, Japan [7-9]. A Cu(001) single crystal was mounted with [110] lying in the horizontal plane, and was cleaned by the repeated cycles of $\mathrm{Ar}^{+}$sputtering at $1.5 \mathrm{keV}$ and subsequent annealing at $\sim 900 \mathrm{~K}$. The clean and ordered $\mathrm{Cu}(001)$ surface was first dosed with $5.2 \times 10^{-4} \mathrm{~Pa} \mathrm{O}_{2}$ for $300 \mathrm{~s}$ at $\sim 500 \mathrm{~K}$. The formation of the well-known $(2 \sqrt{2} \times \sqrt{2}) \mathrm{R} 45^{\circ}$-O reconstruction structure was confirmed by a low-energy electron diffraction (LEED) pattern. The Ni films were then grown at room temperature by the electron bombardment heating of a $\mathrm{Ni}$ rod at a typical growth rate of $0.2 \mathrm{ML} / \mathrm{min}$. The layer-by-layer growth of the Ni films was confirmed and the film thickness was controlled by an in-situ observation of the oscillatory behavior of the reflection highenergy electron diffraction intensity. It has been reported that oxygen stays at the surface during the growth of the $\mathrm{Ni}$ film, and acts as a surfactant to improve the layerby-layer growth of $\mathrm{Ni}$ [10-12]. After these procedures, the $\mathrm{O} / \mathrm{Ni} / \mathrm{Cu}(100)$ sample was obtained, which was confirmed by the $\mathrm{c}(2 \times 2)$-O LEED pattern as reported in the literature [10-12].

The depth-resolved XAFS/XMCD data were taken just after the sample preparation in the same chamber without breaking vacuum. The XMCD spectra were recorded in the grazing incidence configuration, as shown in Fig. 1(c), in which the in-plane magnetization is detected, by using the circular polarization. A pulsed magnetic field of $\sim 500$ Oe was applied, which was parallel to the X-ray beam, before each measurement. Since the film shows inplane magnetization at remanence, the sample was magnetized in the [110] direction in the present setup. Here, we define the [110] and [001] directions as $x$ and $z$, respectively.

The X-ray linear dichroism measurement was also performed after the sample was magnetized in the [110] $(x)$ direction. The horizontal and vertical linear polarizations were used in the normal incidence configuration, as shown in Fig. 1(b), in order to make the electric vector, $\boldsymbol{E}$, parallel and perpendicular to the [110] direction, respectively, denoted as $\boldsymbol{E} / / x$ and $\boldsymbol{E} / / y$. On the other hand, the grazing incidence configuration was adopted with horizontal linear polarization in order to make $\boldsymbol{E}$ nearly parallel to the [001] direction, denoted as $\boldsymbol{E} / / \sim z$.

\section{RESULTS AND DISCUSSION}

Figure 2(a) shows Ni L-edge XAFS spectra for $\mathrm{O} / \mathrm{Ni}(5.5 \mathrm{ML}) / \mathrm{Cu}(001)$ taken at $\sim 100 \mathrm{~K}$ in the $\boldsymbol{E} / / x$ configuration with different $\lambda_{\mathrm{e}}$ ranging from 0.6 to $1.0 \mathrm{~nm}$. All the spectra show basically the same feature, but one can find an increase in the $\mathrm{L}_{3}$ peak intensity at $\sim 852 \mathrm{eV}$ with decreasing $\lambda_{\mathrm{e}}$, i.e. increasing surface sensitivity, as
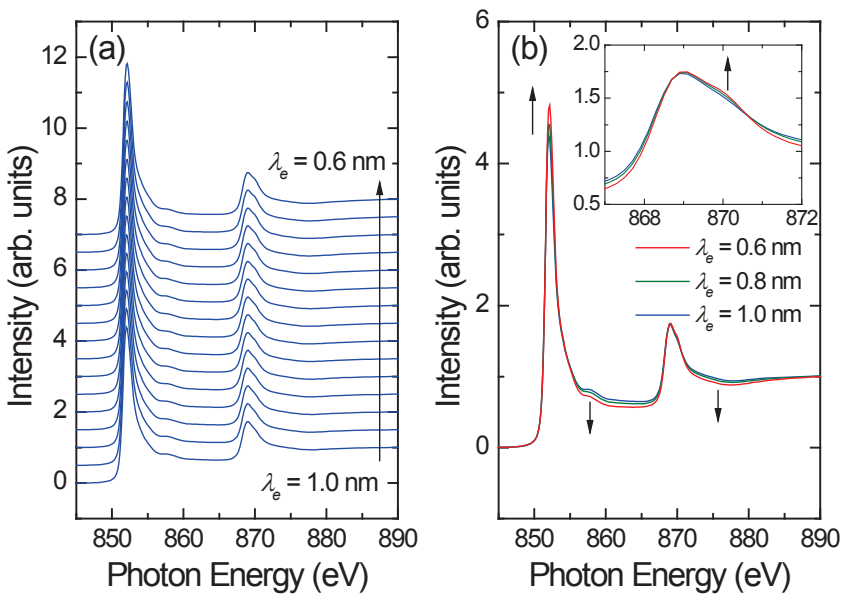

FIG. 2: Ni L-edge XAFS data for $\mathrm{O} / \mathrm{Ni}(5.5 \mathrm{ML}) / \mathrm{Cu}(001)$ taken at different $\lambda_{\mathrm{e}}$ ranging from 0.6 to $1.0 \mathrm{~nm}$, corresponding to the electron detection angles, $\theta_{\mathrm{d}}$, of $\sim 2-16^{\circ}$ (a) and those at $\lambda_{\mathrm{e}}=0.6,0.8$, and $1.0 \mathrm{~nm}$ (b).
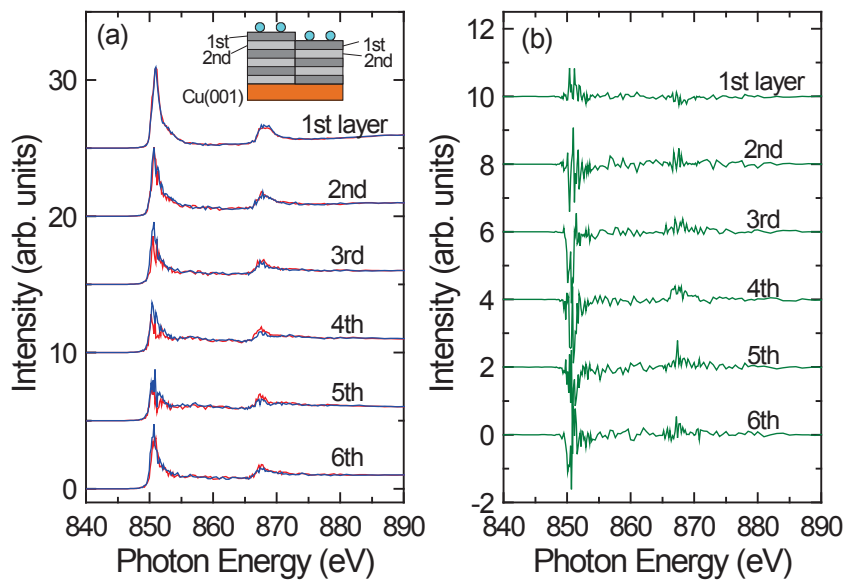

FIG. 3: Ni L-edge X-ray absorption spectra for circular polarizations (a) and their difference (b) at each Ni layer of the $\mathrm{O} / \mathrm{N}(5.5 \mathrm{ML}) / \mathrm{Cu}(001)$ film extracted from a set of spectra at different $\lambda_{\mathrm{e}}$. The Ni layers are assumed to consist of two parts with the same ratio, in which the thicknesses are 5 and $6 \mathrm{ML}$, and the layer number is counted from the top layer as illustrated.

indicated by an arrow in Fig. 2(b). A decrease in the plateau intensity above the $\mathrm{L}_{3}$ and $\mathrm{L}_{2}$ peaks is also found, as well as that in the satellite feature appearing at a $6 \mathrm{eV}$ higher photon energy than the $\mathrm{L}_{3}$ main peak. Moreover, a shoulder structure at the higher energy side of the $\mathrm{L}_{2}$ peak $(\sim 870 \mathrm{eV})$ becomes more prominent at smaller $\lambda_{\mathrm{e}}$, as shown in the inset of Fig. 2(b).

From a set of XAFS/XMCD data with different $\lambda_{\mathrm{e}}$, the layer-resolved spectra are extracted. In the extraction process, it is assumed that the $5.5 \mathrm{ML} \mathrm{Ni}$ film consists of two parts with the same ratio, in which the thicknesses are 5.0 and 6.0 ML. The layer-resolved XAFS/XMCD spectra are determined so as to reproduce the observed spectra with different $\lambda_{\mathrm{e}}$ according to Eq. (1), and are shown in Fig. 3. The surface component exhibits characteristic spectral features of $\mathrm{NiO}[13]$, indicating that a $\mathrm{NiO}-$ like layer is formed at the surface of the $\mathrm{Ni}$ film. On the other hand, the spectra for the underlying layers look 

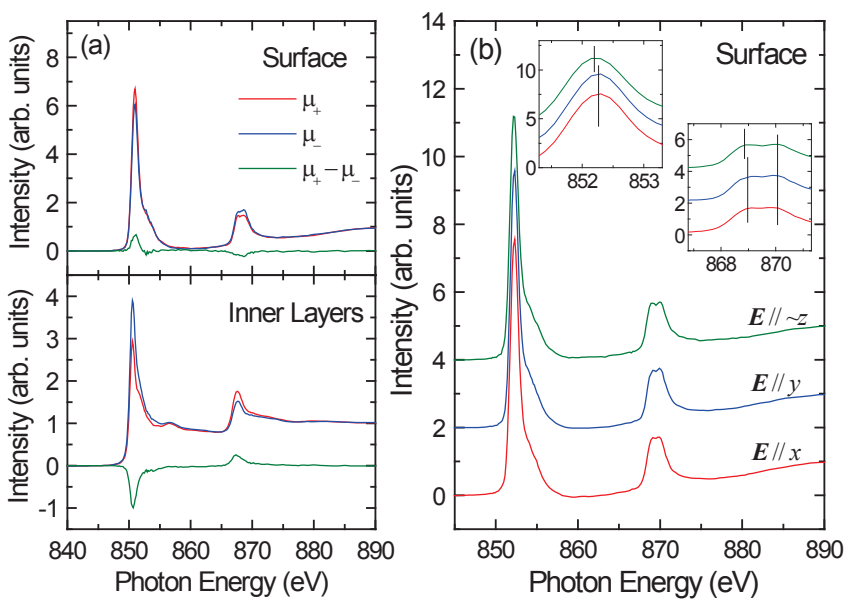

FIG. 4: Ni L-edge X-ray absorption spectra for circular polarizations $\left(\mu_{+}\right.$and $\left.\mu_{-}\right)$and their difference $\left(\mu_{+}-\mu_{-}\right)$at the surface and in the inner layers (a) and those at the surface layer for three different linear polarizations (b) extracted by assuming a two-region model.

like that of a typical metallic Ni film, suggesting that the inner $\mathrm{Ni}$ layers are not oxidized, which is reasonable because the oxygen atom always stays at the surface [10-12]. Moreover, it should be emphasized here that the XMCD spectrum at the surface has an opposite sign with respect to those in the underlying layers. Therefore, we hereafter adopt a two-region model, in which the Ni layers are divided into a surface single layer and remaining inner layers.

The XMCD spectra at the surface and in the inner layers are shown in Fig. 4(a). The inner layers exhibit a typical XMCD spectrum for a Ni metal film. The estimated spin magnetic moment is $0.54 \pm 0.5 \mu_{\mathrm{B}} /$ atom, which is similar to that for bulk Ni. In contrast, the XMCD spectrum for the surface layer exhibits an opposite sign to that of the inner layers, and the estimated magnetic moment is $-0.3 \pm 0.2 \mu_{\mathrm{B}}$ /atom. This suggests the existence of an uncompensated spin moment in the surface NiO-like layer, which has an antiparallel magnetic coupling to the inner ferromagnetic Ni layers.

Finally, let us discuss the X-ray linear dichroism of the surface NiO-like layer. The extracted X-ray absorption spectra at different X-ray incidence conditions are plotted in Fig. 4(b). Since the $x$ and $y$ axes are equivalent considering the fourfold symmetry of the (001) surface except for the magnetic structures, the X-ray magnetic linear dichroism (XMLD) between the $\boldsymbol{E} / / x$ and $\boldsymbol{E} / / y$ configurations is sensitive to the spin direction of the NiO-like layer. No significant difference is found, however, indicating that the spin moment is not aligned in either $x$ or $y$ direction. On the other hand, small peak shifts are found between the in-plane $(\boldsymbol{E} / / x$ and $\boldsymbol{E} / / y)$ and out-of-plane $(\boldsymbol{E} / / \sim z)$ configurations. This dichroic behavior is consistent with the linear dichroism, which is not attributed to the antiferromagnetic order but to the crystal-field effect due to the low symmetry of the $1 \mathrm{ML}$ NiO-like layer [13]. Therefore, we conclude that no magnetic order can be detected in the NiO-like layer, at least in the primitive directions, $x, y$, and $z$.

The obtained results, a small XMCD signal in the [110] $(x)$ direction and no XMLD between $x$ and $y$ directions at the surface NiO-like layer, would be interpreted as follows: The surface NiO-like layer has an antiferromagnetic order with two equivalent domains, in which the spin moment is aligned between the $x$ and $y$ directions, but slightly inclined via the magnetic interaction with the underlying Ni layers [6]. In such case, the XMLD signal is canceled out by averaging over the domains, but a small XMCD signal appears.

\section{CONCLUSIONS}

We have presented the capability of the depth-resolved XAFS/XMCD technique to observe the surface and interface spectra with an atomic-layer resolution. As an example, we have applied the technique to an oxygen-covered $\mathrm{Ni}$ film, and revealed the formation of a 1-ML-thick NiOlike layer at the surface. The XMCD spectra indicate the existence of an uncompensated spin moment in the NiO-like layer, which is antiparallel to the spin moment in underlying Ni layers. In contrast, the XMLD spectra show no preferential direction for the $\mathrm{Ni}$ spin moment in the surface NiO-like layer with respect to the magnetization direction, $x$, of the underlying Ni layers. These results would be interpreted by assuming that the NiOlike layer has an antiferromagnetic order with two equivalent domains, in which the spin moment is inclined by $45^{\circ}$ from the $x$ direction, but slightly rotates via the magnetic interaction with the underlying Ni layers. We have thus demonstrated a possibility to investigate chemical state and magnetic structure at the atomic-level surface and interface by using the depth-resolved XAFS/XMCD technique.

\section{Acknowledgments}

The authors are grateful for the financial support of the Quantum Beam Technology Program from the Ministry of Education, Culture, Sports, Science and Technology.
[1] B. T. Thole, P. Carra, F. Sette, and G. van der Laan, Phys. Rev. Lett. 68, 1943 (1992).

[2] P. Carra, B. T. Thole, M. Altarelli, and X. Wang, Phys. Rev. Lett. 70, 694 (1993).

[3] K. Amemiya, S. Kitagawa, D. Matsumura, T. Yokoyama, and T. Ohta, J. Phys.: Condens. Matter 15, S561 (2003).

[4] K. Amemiya, S. Kitagawa, D. Matsumura, H. Abe, T.
Ohta, and T. Yokoyama, Appl. Phys. Lett. 84, 936 (2004).

[5] K. Amemiya, Phys. Chem. Chem. Phys. 14, 10477 (2012).

[6] K. Amemiya and M. Sakamaki, Appl. Phys. Lett. 98, 012501 (2011).

[7] K. Amemiya, H. Kondoh, T. Yokoyama, and T. Ohta, J. Electron Spectrosc. Relat. Phenom. 124, 151 (2002).

[8] Y. Kitajima, K. Amemiya, Y. Yonamoto, T. Ohta, T. 
Kikuchi, T. Kosuge, A. Toyoshima, and K. Ito, J. Synchrotron Rad. 5, 729 (1998).

[9] K. Amemiya, A. Toyoshima, T. Kikuchi, T. Kosuge, K. Nigorikawa, R. Sumii, and K. Ito, AIP Conf. Proc. 1234, 295 (2010)

[10] R. Nunthel, T. Gleitsmann, P. Poulopoulos, A. Scherz, J. Lindner, E. Kosubek, Ch. Litwinski, Z. Li, H. Wende, and K. Baberschke, Surf. Sci. 531, 53 (2003).
[11] J. Lindner, P. Poulopoulos, R. Nünthel, E. Kosubek, H. Wende, and K. Baberschke, Surf. Sci. 523, L65 (2003).

[12] J. Hong, R. Q. Wu, J. Lindner, E. Kosubek, and K. Baberschke, Phys. Rev. Lett. 92, 147202 (2004).

[13] M. W. Haverkort, S. I. Csiszar, Z. Hu, S. Altieri, A. Tanaka, H. H. Hsieh, H.-J. Lin, C. T. Chen, T. Hibma, and L. H. Tjeng, Phys. Rev. B 69, 020408(R) (2004). 